\title{
Allgemeinbildung im Informationszeitalter?
}

Der tschechische Schriftsteller Karel Capek veröffentlichte 1920 ein sozialutopisches Drama mit dem Titel Rossum's Universal Robots. Die darin vorkommenden selbstbeweglichen Automaten, die mehr oder weniger der menschlichen Gestalt nachgebildet waren, nannte er in Anlehnung an das tschechische Wort "robota" für Fronarbeit "Roboter". Der Roman erwies sich in mehreren Sprachen als Bestseller und der Begriff "Roboter" war geboren.

Am Beispiel von Spielzeugrobotern, genauer endlichen Automaten, und deren Programmierung stellt J. Nievergelt seine Gedanken und Argumente für eine zeitgemäße Allgemeinbildung, die die Informatik einbezieht, zur Diskussion.

Heribert Vollmer stellt die Frage "Was leistet die Komplexitätstheorie für die Praxis?" und mischt sich damit in eine Diskussion ein, die zur Zeit inner- und außerhalb der Theoretischen Informatik intensiv geführt wird. Der Autor demonstriert an verschiedenen Beispielen, wie die oft als praxisfremd angesehene Komplexitätstheorie zumindest Orientierungshilfe, oft aber auch unmittelbar anwendbare Antworten auf Fragen von praktischer Bedeutung geben kann.

Aus der allgegenwärtigen Anwendung schlüsselfertiger Software folgt nicht, daß Programmierkenntnisse nur von professionellen Programmierern gelernt werden müssen. Zeitliche Abläufe korrekt zu spezifizieren, sollte zur Allgemeinbildung gehören, analog den mathematischen Beweisen, deren Ergebnisse man täglich anwendet, obwohl man die in der Schule oder im Studium gelernten Beweismethoden im allgemeinen nicht heranzieht.

Dem sich in stürmischer Entwicklung befindlichen Thema Electronic Commerce sind zwei Beiträge aus unterschiedlichen Blickwinkeln gewidmet. M. Merz, Tuan Tu und W. Lamersdorf betrachten die wesentlichen EC-Mechanismen und geben einen Überblick über relevante Standards, Zahlungsverfahren und Systemarchitekturen. Die Integration von Softwareagenten in elektronische Märkte untersuchen R. Zarnenkow und W. Brenner.

Das aktuelle Schlagwort befaßt sich mit einem weiteren Brandthema, der Bioinformatik, zu dem Sie im Forum auch einen programmatischen Beitrag „Bioinformatik $2000^{\prime \prime}$ von R. Hofestädt finden.

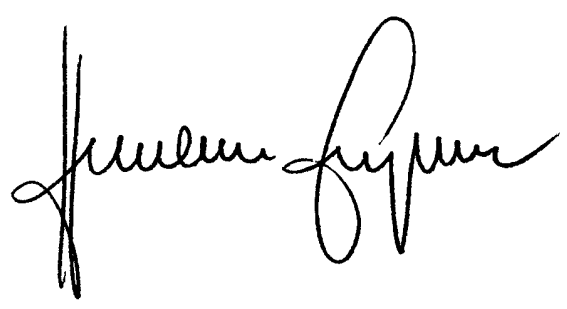

